\title{
Osteoid osteoma: treatment outcome and long-term follow-up after MRI-guided laser ablation
}

\author{
Ricarda Seemann $^{1 \#} \wedge$, Georg Böning ${ }^{2 \#}$, Philipp Schwabe ${ }^{3}$, Ulf Teichgräber ${ }^{4}$, Bernhard Gebauer ${ }^{2}$, \\ Florian Streitparth ${ }^{5}$
}

${ }^{1}$ Centre for Musculoskeletal Surgery, Charité - University Medicine Berlin, Corporate Member of Freie Universität Berlin, Humboldt-Universität zu Berlin, and Berlin Institute of Health, Berlin, Germany; ${ }^{2}$ Department of Radiology, Charité - University Medicine Berlin, Corporate Member of Freie Universität Berlin, Humboldt-Universität zu Berlin, and Berlin Institute of Health, Berlin, Berlin, Germany; ${ }^{3}$ Department for Motion Surgery, Vivantes Clinic Spandau, Berlin, Germany; ${ }^{4}$ Department of Radiology, University Hospital, Friedrich Schiller University Jena, Jena, Germany; ${ }^{5}$ Department of Radiology, University Hospital, LMU Munich, Munich, Germany

Contributions: (I) Conception and design: All authors; (II) Administrative support: R Seemann, G Böning; (III) Provision of study materials or patients: R Seemann, G Böning, P Schwabe, F Streitparth; (IV) Collection and assembly of data: R Seemann, P Schwabe, F Streitparth; (V) Data analysis and interpretation: R Seemann, U Teichgräber, B Gebauer, F Streitparth; (VI) Manuscript writing: All authors; (VII) Final approval of manuscript: All authors.

\#These authors contributed equally to this work.

Correspondence to: Ricarda Seemann. Centre for Musculoskeletal Surgery, Charité - Universitätsmedizin Berlin, Augustenburger Platz 1, 3353 Berlin, Germany. Email: ricarda.seemann@charite.de.

Background: Aim of this study was to investigate short-term and long-term treatment outcome, complication rates, and patient satisfaction after MRI-guided laser ablation (LA) of osteoid osteoma (OO).

Methods: Thirty-five patients with OO in typical and atypical localizations were treated by MRI-guided LA with MRI thermometry in an open $1.0 \mathrm{~T}$ system. Twenty-nine patients underwent a standardized telephone interview including questions about recurrence, residual pain or functional symptoms, and satisfaction for short-term follow-up after a mean of 31 months. Twenty-one of these patients were available for long-term telephone follow-up after a mean of 116 months.

Results: Technical success of MRI-guided LA was $100 \%$ without major complications. Two minor complications included transient local inflammation and transient damage of the peroneal nerve associated with improper patient positioning during the procedure. Primary clinical success was $92 \%(11 / 12)$ in typically located OO and $82 \%(14 / 17)$ in atypically located OO. Secondary clinical success after repeat ablation was $100 \%$ regardless of OO location. Patient satisfaction and acceptance of the intervention were very good at both short-term (97\%) and long-term (100\%) follow-up.

Conclusions: MRI-guided LA of OO is a safe and effective treatment option resulting in high short-term and long-term patient satisfaction and acceptance rates. Recurrence and adverse events were more common in patients with atypically located OO. Level of Evidence: Level 3, non-randomized follow-up study.

Keywords: Osteoid osteoma (OO); interventional MRI; interventional radiology; thermal ablation; long-term outcome

Submitted Jun 28, 2021. Accepted for publication Oct 12, 2021.

doi: $10.21037 / \mathrm{atm}-21-3343$

View this article at: https://dx.doi.org/10.21037/atm-21-3343

^ ORCID: 0000-0001-5752-070X. 


\section{Introduction}

Osteoid osteoma (OO) is a benign osteoblastic neoplasm that usually occurs in the first three decades of life and commonly causes nocturnal pain responding to nonsteroidal anti-inflammatory drugs (NSAID) such as acetylsalicylic acid (1-3). OO most frequently affects long bones like the femur or tibia. In contrast to this "typical" localization, OO are referred to as "atypical" or "technically challenging" when found in spinal column, shoulder girdle, and pelvis (trunk skeleton) or small bones of the distal extremities (2,4-6).

In the last decades, treatment of OO has shifted towards minimally invasive methods such as image-guided thermal ablation $(7,8)$. Open surgical resection plays a subordinate role $(8,9)$. With regard to nonsurgical focal therapies for OOs, several options exist. Mainly due to its wide availability and familiarity, CT-guided radiofrequency ablation (RFA) has become the treatment of choice with very good success rates, few complications, and high patient satisfaction in several clinical studies (6,10-15). However, as an alternative for image guidance, MRI offers potential advantages, such as better visualization of structures at risk and real-time temperature monitoring during thermal procedures $(16,17)$. In addition, various primary thermal ablation procedures exist. RFA has been used the longest, and more recently microwave ablation (MWA) and cryoablation have also been used, each with specific advantages and disadvantages (18). Combined with MRI-guidance, high intensity focused ultrasound (HIFU) also offers an alternative, especially in children $(19,20)$.

In this study, the combination of MRI guidance and laser ablation (LA) was investigated which offers possible advantages, e.g., MRI and laser have no interferences enabling online MR thermometry or laser showing cost advantages over RFA (21). Little data is available on MRI guidance and the potential of MRI thermometry during LA in the treatment of $\mathrm{OO}(7,22,23)$. In addition, most studies have been conducted with relatively short followup periods (14). The aim of the present study was to fill this gap and to investigate MRI-guided LA of OO in terms of treatment outcome, complications, and patient satisfaction at short-term and long-term follow-up.

We present the following article in accordance with the MDAR reporting checklist (available at https://atm. amegroups.com/article/view/10.21037/atm-21-3343/rc).

\section{Methods}

\section{Study population}

This was a retrospective single-center study. The study was conducted in accordance with the Declaration of Helsinki (as revised in 2013). The study was approved by Institutional Ethics Committee of Charité - University Medicine Berlin (No. EA1/301/12) and individual consent for this retrospective analysis was waived. Thirty-five patients with diagnosed $\mathrm{OO}$ were treated by MRI-guided LA in our clinic between June 2008 and July 2013.

Twenty-nine patients took part in a standardized telephone interview for short-term follow-up after a mean of $31 \pm 14$ months ( 3 -year follow-up) and were included in this study. Twenty-one of those patients were available for a second telephone interview for long-term followup after a mean of $116 \pm 13$ months (10-year follow-up). Of the initial 35 patients, 6 were lost to short-term followup, and another 8 were lost to long-term follow-up (one patient died of a cause unrelated to the study, 7 moved to an unknown address/did not give consent) (see Figure 1).

Primary endpoints included technical success (defined as interventional procedure successfully executed with the applicator reaching the target position with MRI guidance) as well as primary and secondary clinical success (defined as freedom from pain and/or residual symptoms after single or repeated ablation). Secondary endpoints were adverse events, patient satisfaction, and acceptance of the procedure.

\section{Interventional procedure}

The interventional procedure was described in detail earlier. For photographic documentation of interventional setup, see Figure 2. The interventions were performed under general and local anesthesia (cutaneous and periosteal) in an open 1.0T MRI system (Panorama HFO, Philips, Best, Netherlands) for MRI-guided percutaneous LA. Before the intervention, a single-shot intravenous antibiotic $(1.5 \mathrm{~g}$ cefuroxime) was administered for infection prophylaxis. The MRI-guided LA technique consists of three main steps: (I) Multiplanar real-time MRI guidance: for realtime interactive lesion localization, instrument guidance, drilling, and positioning of the laser fiber within the lesion, a fast T1-weighted turbo spin echo (TSE) sequence [echo time (TE)/repetition time (TR) 5.7/200 ms, turbo factor (TF) 7, flip angle (FA) $90^{\circ}$, scan duration $3 \mathrm{~s}$ ] was acquired. Full anatomical orientation was achieved by multiplanar 


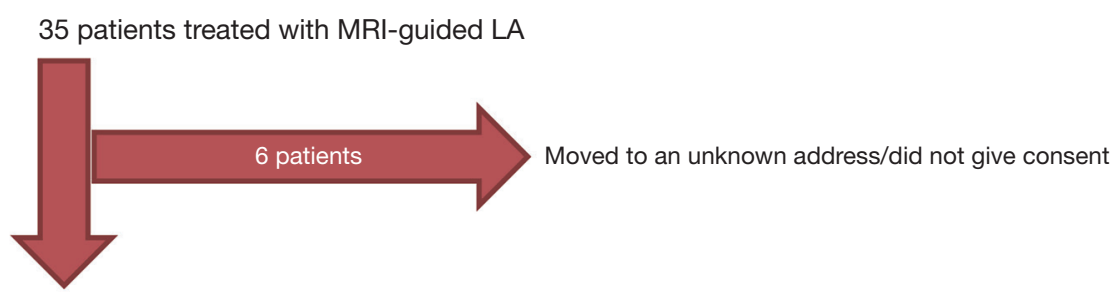

29 patients with 3-year follow-up

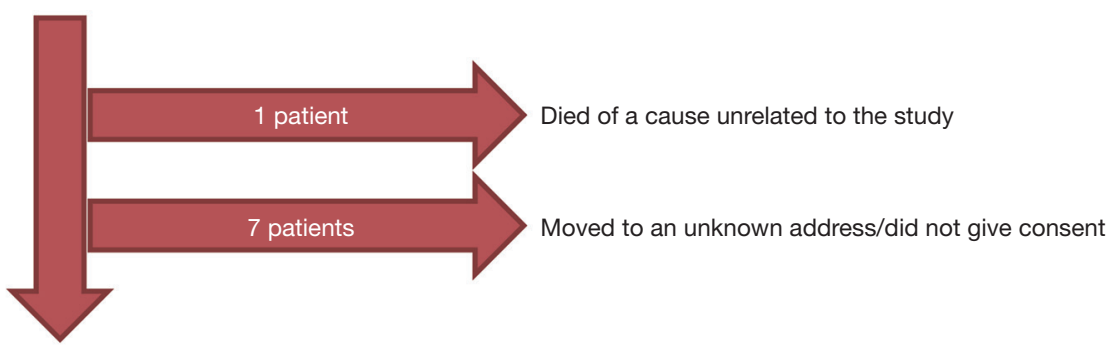

21 patients with 10-year follow-up

Figure 1 Patients included for follow-up. LA, laser ablation.
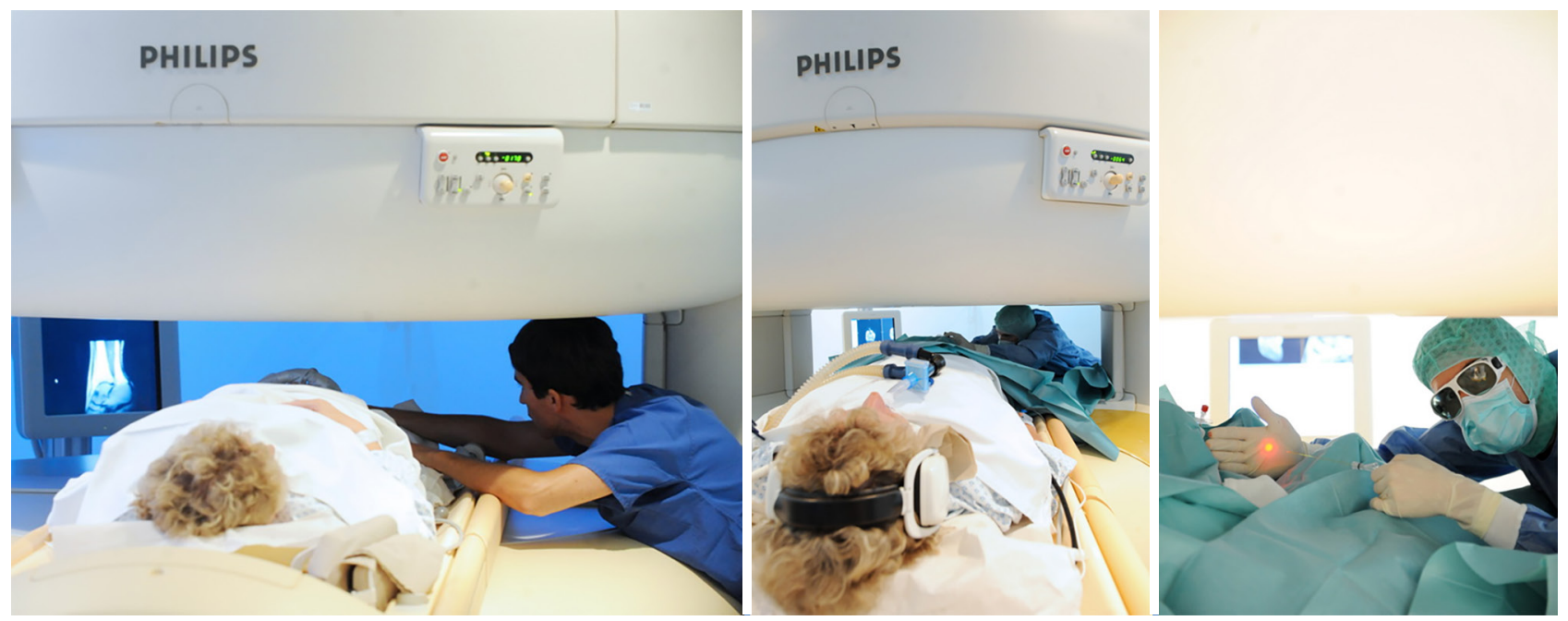

Figure 2 View of the interventional set up in the MRI scanner room. A 64-year-old female patient with OO of the right talus as presented in Figure 3. The patient is positioned in the open high-field MRI with lower extremity placed in the magnet's iso-center. Real-time MRI guidance ensures correct introduction of the laser probe. Note additional monitoring of instrument positioning and laser release during intervention. OO, osteoid osteoma.

imaging [including (para-)axial, (para-)sagittal, and (para-) coronal]. Depending on the extent of perifocal ossification and localization of the target lesion, an MRI-compatible bone biopsy drill (Invivo, Schwerin, Germany) was used to prepare the puncture tract before a $16-18 \mathrm{G}$ needle (Somatex, Teltow, Germany) was introduced. A proton density-weighted (PDw) TSE sequence (TE/TR 30/383 ms, $\mathrm{TF} 11, \mathrm{FA} 90^{\circ}$, scan duration $41 \mathrm{~s}$ ) in two planes was used to verify correct needle positioning. (II) LA with online MRI thermometry: after introduction of a $600 \mu \mathrm{m}$ bare laser fiber (Frank Optic Products ${ }^{\circledR}$, Berlin, Germany), a Nd: YAG laser (1,064 nm, Fibertom Medilas, Dornier MedTech, Wessling, 
Table 1 Patient characteristics and localizations of $\mathrm{OO}$

\begin{tabular}{|c|c|}
\hline Patient characteristics & Data \\
\hline Patients treated with MR-guided LA (n) & 35 \\
\hline Patients included in follow-up (n) & 29 \\
\hline Sex $(w ; m)$ & $9 ; 20$ \\
\hline Age (years), median (range; IQR) & $24(4-64 ; 22)$ \\
\hline Follow-up 1 ( $n=29)$ (months), mean $\pm S D$ & $31 \pm 14$ \\
\hline Follow-up $2(n=21)$ (months), mean $\pm S D$ & $116 \pm 13$ \\
\hline Localization (typical; atypical) & $12 ; 17$ \\
\hline \multicolumn{2}{|l|}{ Upper extremity $(n=2)$} \\
\hline Humerus & 1 \\
\hline Finger & 1 \\
\hline \multicolumn{2}{|l|}{ Lower extremity $(n=25)$} \\
\hline Femoral neck & 5 \\
\hline Femoral condyle & 1 \\
\hline Femur & 4 \\
\hline Patella & 1 \\
\hline Tibial head & 2 \\
\hline Tibia & 6 \\
\hline Fibula & 1 \\
\hline Talus & 2 \\
\hline Heel bone & 1 \\
\hline Metatarsal & 1 \\
\hline Toe & 1 \\
\hline Acetabulum & 1 \\
\hline Iliac bone & 1 \\
\hline
\end{tabular}

OO, osteoid osteoma; MR, magnetic resonance; LA, laser ablation; IQR, interquartile range.

Germany) with continuous energy flow and an effective output ranging from 2 to $3 \mathrm{~W}$ was used for subsequent treatment. Total energy deposition ranged from 360-4,300 $\mathrm{J}$ depending on target lesions size and localization. Online MRI thermometry was used for monitoring the temperature tissue effects utilizing a T1w gradient echo (GRE) sequence (TE/TR 2/4.3 ms; FA $27^{\circ}$ ) with $4 \mathrm{~s}$ update time ensuring complete and safe ablation.

(III) Post-interventional control MRI: contrast-enhanced sequences (T1w SE sequences (TE/TR 12/503 ms, FA 90, TA $92 \mathrm{~s}$ ) and subtraction images were used to verify therapy efficacy directly after the intervention based on signal loss in the nidus. Contrast agent (Gadovist; Bayer- Schering, Berlin, Germany) injection followed a weight-based protocol $(0.1 \mathrm{~mL} / \mathrm{kg}$ body weight).

All patients were observed to identify possible postinterventional complications, such as neurovascular damage, bleeding, or burns, before discharge from hospital within $48 \mathrm{~h}$ after the procedure.

\section{Follow-up Imaging and Patient Interviews}

To compare the extent of the ablation area with the size of the former target lesion and to detect any residual/ recurrent contrast enhancement of the nidus, control imaging was performed at intervals of 3,6 , and 12 months. The standard protocol included fat-saturated $\mathrm{T} 2$-weighted TSE sequences (SPIR, TE/TR 60/1,600 ms, FA 90 ${ }^{\circ}$ ) and contrast-enhanced sequences with subtraction images as aforementioned (24).

Short-term and long-term follow-up was performed by a structured telephone interview with the following items: clinical success (recurrence or not?), residual pain, patient satisfaction, and willingness to undergo the procedure again if necessary and indicated. The interview questionnaire was modified from Seemann et al. (6).

\section{Statistical analysis}

Descriptive statistical evaluation was performed with Excel (Microsoft Inc., Redmond, WA, USA) and SPSS v.26 (IBM, Armonk, NY, USA). We report absolute and relative frequencies for categorical variables and used the ShapiroWilk test to test for normal distribution. According to the results, mean and standard deviation (SD) are given for normally distributed interval scaled data and median, interquartile range (IQR), and range for nonnormally distributed, interval-scaled data.

\section{Results}

\section{Patient characteristics (Table 1)}

Follow-up data were collected from 29 patients, among them 9 women and 20 men (female-to-male sex ratio of 9:20). Median age at the time of intervention was 24 years (youngest patient four years, oldest patient 64 years). Twelve patients ( $41 \%$ ) had OO in typical localization, whereas 17 patients (59\%) had atypical OO. A very large 
Table 2 Technical success (defined as interventional procedure successfully executed with the applicator reaching the target position with MRI guidance) and clinical success (defined as freedom from pain and/or residual symptoms after single or repeated ablation), adverse events, and patient satisfaction

\begin{tabular}{ll}
\hline Endpoints & Percentage \\
\hline Primary endpoints & \\
Technical success & $100 \%$ \\
Primary clinical success & $86 \%$ (switch to open surgery $\mathrm{n}=2)$ \\
Secondary clinical success & $100 \%$ \\
Secondary endpoints & \\
Adverse events & Minor $\mathrm{n}=2$ (1 transient local \\
& inflammation, 1 transient nerval \\
& lesion) \\
& Major $\mathrm{n}=0$ \\
Patient satisfaction & $97 \%$ after 3 years \\
& $100 \%$ after 10 years \\
\hline
\end{tabular}

majority of patients, $86 \%(25 / 29)$, had OO of the lower extremity. The tibia was the most common site of $\mathrm{OO}$ in the legs $(6 / 25)$. Localization of OO in the upper extremity $(2 / 29)$ or trunk skeleton $(2 / 29)$ was rare in our patient population.

\section{Technical and clinical success, adverse events, and patient satisfaction (Table 2)}

Technical success (defined as interventional procedure successfully executed with the applicator reaching the target position with MRI guidance) of MRI-guided LA was $100 \%$. Primary clinical success (defined as freedom from pain and/or residual symptoms after single ablation) was $86 \%(25 / 29)$. For one patient with persistent pain after the first intervention who opted to be treated in a different clinic, no information on outcome was available. Three of the 29 patients $(10 \%)$ had recurrence. All three recurrent OOs were located atypically. Two of these were located in the femoral neck; one of the patients underwent a second MRI-guided LA, the other underwent CT-guided RFA. The third patient with recurrence had OO located in the toe and underwent open surgery. All three patients were free of symptoms afterwards. Secondary clinical success in the patients who underwent repeat MRI-guided LA was therefore $100 \%$.

There were no major complications, and two minor adverse events occurred, both in atypically located OO: one patient with $\mathrm{OO}$ in the distal phalanx of digit IV experienced a transient local inflammatory reaction. One patient with OO located in the proximal femur suffered a transient lesion of the peroneal nerve most likely attributable to malpositioning and pressure on the nerve during the procedure, which resolved over time. Altogether, recurrence of $\mathrm{OO}$ or primary clinical failure as well as development of adverse events were more likely to occur in patients with atypical OO. For illustration, see Figure 3 and Figure 5 for atypical location of OO, Figure 4 for typical location, and Video 1 showing online MRI thermometry.

The follow-up interviews revealed a high level of patient satisfaction and acceptance of the procedure both at shortterm follow-up (97\% of patients satisfied) and long-term follow-up (100\% of patients satisfied). The readiness to undergo MRI-guided LA again if necessary was stated by $97 \%$ at short-term follow-up and by all patients (100\%) interviewed at long-term follow-up.

\section{Discussion}

OO treatment strategies have changed over the years, and image-guided local ablative procedures are now the method of choice $(14,25)$. Besides cryoablation, hyperthermal techniques such as RFA, LA and MWA allow precise tumor ablation with high success rates while being less invasive and having fewer complications than open surgery $(6,10,13,14,26,27)$.

Although there are cost disadvantages compared with LA (21), RFA is commonly used (14). CT is still the most common imaging modality for puncture guidance in minimally invasive OO treatment (28), but MRI guidance has also proven to be feasible in open or wide-bore MRI scanners, providing adequate patient access as well as highquality imaging for lesion localization and instrument guidance (7). Further advantages of MRI include multiplanar navigation capabilities, online temperature monitoring during treatment (MRI thermometry), and immediate postinterventional verification of treatment success $(6,7,24,29,30)$.

CT navigation for ablations is more widely available compared with MRI and has cost advantages. However, since OOs are regularly treated in younger patients, radiation exposure should be kept as low as possible in accordance with the ALARA (as low as reasonably achievable) principle. Especially if $\mathrm{OO}$ occur in the pelvic skeleton, radiation exposure resulting from CT guidance 

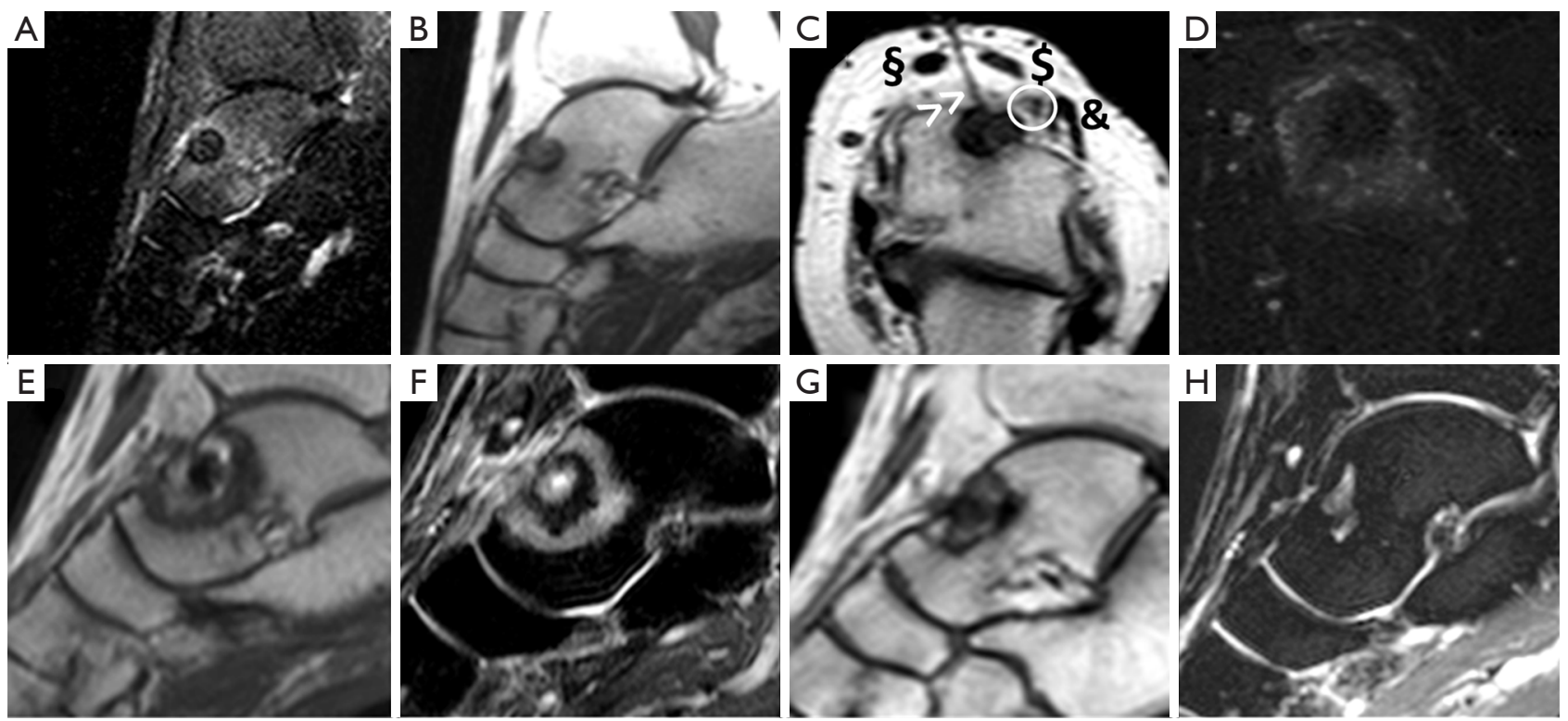

Figure 3 A 64-year-old female patient with $\mathrm{OO}$ of the right talus (atypical localization) presenting with pain associated with weight-bearing. Technical and clinical success, no complications. Preprocedural SPIR (A) and T1w (B) sequences in sagittal planes show osteoid osteoma of the talus. Periprocedural verification of instrument placement in axial plane (C). Immediate postablational subtraction imaging shows no residual nidal enhancement while showing a reactive perinidal enhancing rim (D). Follow-up imaging reveals typical target-sign appearance 3 months after the procedure in T1w (E) and SPIR (F) images. Further follow-up imaging reveals shrinkage of the postablational lesion and its different zonal compartments after 6 months ("positive inward fusion"), (G). Thirty-six months after the procedure, zonal differentiation has vanished; an irregularly shaped, hyperintense signal most likely represents residual scar tissue in the SPIR sequence (H). ${ }^{>}$, needle; $\$$, anterior tibial tendon; ${ }^{\$}$, tendon of extensor hallucis longus muscle; ${ }^{*}$, tendon of extensor digitorum longus muscle; ${ }^{\circ}$, peroneus profundus nerve and dorsal pedis artery. OO, osteoid osteoma; T1w, T1 weighted; SPIR, spectral presaturation with inversion recovery.

is a concern even when state-of-the-art techniques with dose reduction (e.g., iterative reconstruction) or cone-beam CT are used $(31,32)$. MRI guidance therefore might be a possible alternative as suggested by experience from MRIguided HIFU $(33,34)$. Compared with MRI-guided HIFU, MRI-guided percutaneous LA has the advantage of shorter treatment duration $(14,33)$. On the other hand, HIFU is an entirely noninvasive method (34). Yet, in our study we did not see any wound healing disorders or infections after LA, underlining that LA of OO is safe. Furthermore, HIFU may have potential limitations like incomplete ablation or collateral damage due to movement of the target region as well as interactions or even reflections of HIFU waves by gas and bone, which may cause adverse effects (35). Both techniques-MRI-guided HIFU and MRI-guided LA of OO can be performed using real-time MRI thermometry, which allows precise monitoring of the progress of ablation and thus helps to spare structures at risk, such as nerves and vessels.

The fact that MRI-guided LA offers the possibility of direct postablational image control of outcome ("one-stop shop") can be considered another advantage of the method. Recently, dynamic contrast-enhanced MRI has also been described as a useful tool for visualization of short-term healing following thermal ablation of OO (36-38). Despite the wide range of imaging options, however, the long-term response to therapy has to be evaluated by clinical followup and patient-reported outcome measures, in particular subjective pain (39).

In this study, the primary clinical success of MRIguided LA was $86 \%$, and $100 \%$ in patients who underwent a second intervention, which is consistent with published results $(6,10)$. In a systematic review, Shanmugasundaram et al. came to a calculated clinical success rate of $94.2 \%$ for LA, yet in these studies only $32 \%$ of OO were located atypically (14). In our study, recurrence of OO or primary 

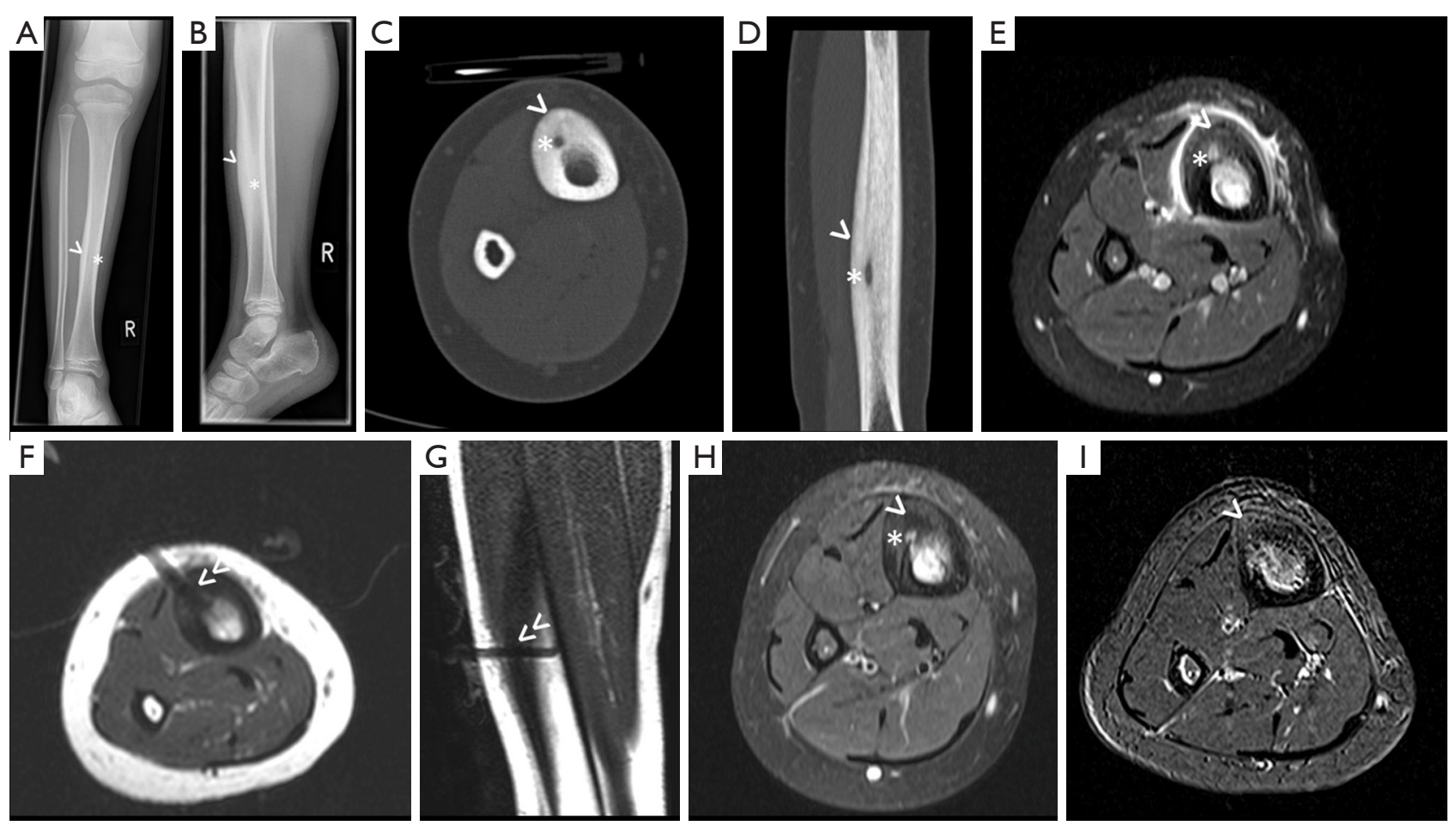

Figure 4 Seven-year-old boy with OO of the right tibial shaft (typical localization) presenting with nocturnal pain. Primary technical and clinical success, no complications. Both conventional X-ray (A,B) and CT scans (C,D) show typical features such as cortical thickening and central nidus. Preprocedural MRI (PDw with fat saturation in axial orientation) (E) visualizes nidus and edema. MRI during intervention with periprocedural verification of instrument placement (PDw TSE in axial (F) and sagittal orientation (G). (H) MRI 3 months after intervention (PDw with fat saturation in axial orientation) showing edema/hematoma in the nidal area; (I) MRI 3 months after intervention (subtraction of contrast-enhanced and native T1w TSE) demonstrating a loss of nidal enhancement, thus proving complete ablation; *, nidus; >>, needle; ', cortical thickening. OO, osteoid osteoma; PDw, proton-density weighted; TSE, turbo spin echo.

clinical failure as well as minor adverse events were most commonly observed in patients treated for atypical OO. This finding is consistent with atypical OOs also being referred to as technically challenging, and may explain the slightly lower primary success rate of $86 \%$ in our patient collective with $59 \%$ atypical OO. In particular in these cases, MRI thermometry may be useful for sparing adjacent neurovascular structures and thus potentially increasing the efficiency and safety of LA in patients with atypical OO. MRI thermometry thus has the potential to improve overall treatment quality. Regarding success and complication rates, our results are comparable with outcomes reported for CT-guided RFA (12,40-42). Lassalle et al., for example, reported a primary success rate of $94.3 \%$ (79/88) and few minor complications (40). Also in line with the results of previous studies, we did not observe any major complications $(6,10,14,43)$.
Atypical OO are also called "technically challenging". One patient with $\mathrm{OO}$ of the toe had to be treated with a modified ablation protocol (low temperature, low duration) in order to save structures at risk (here: close to the skin). He suffered recurrence and underwent open surgery, yet secondary treatment using CT-guided RFA would also have been an option in this location. Another patient with recurrent $\mathrm{OO}$ in difficult location, in the femoral neck, also underwent successful secondary treatment using CT-guided RFA. Due to the ALARA principle regarding radiation exposure, which is especially important in young patients, MRI guidance should be considered as the primary therapy recommendation if available, reserving CT guidance as secondary alternative when higher spatial resolution may be needed for optimal outcome.

Subjective treatment satisfaction in our patients was very good at both short-term (97\%) and long-term (100\%) 

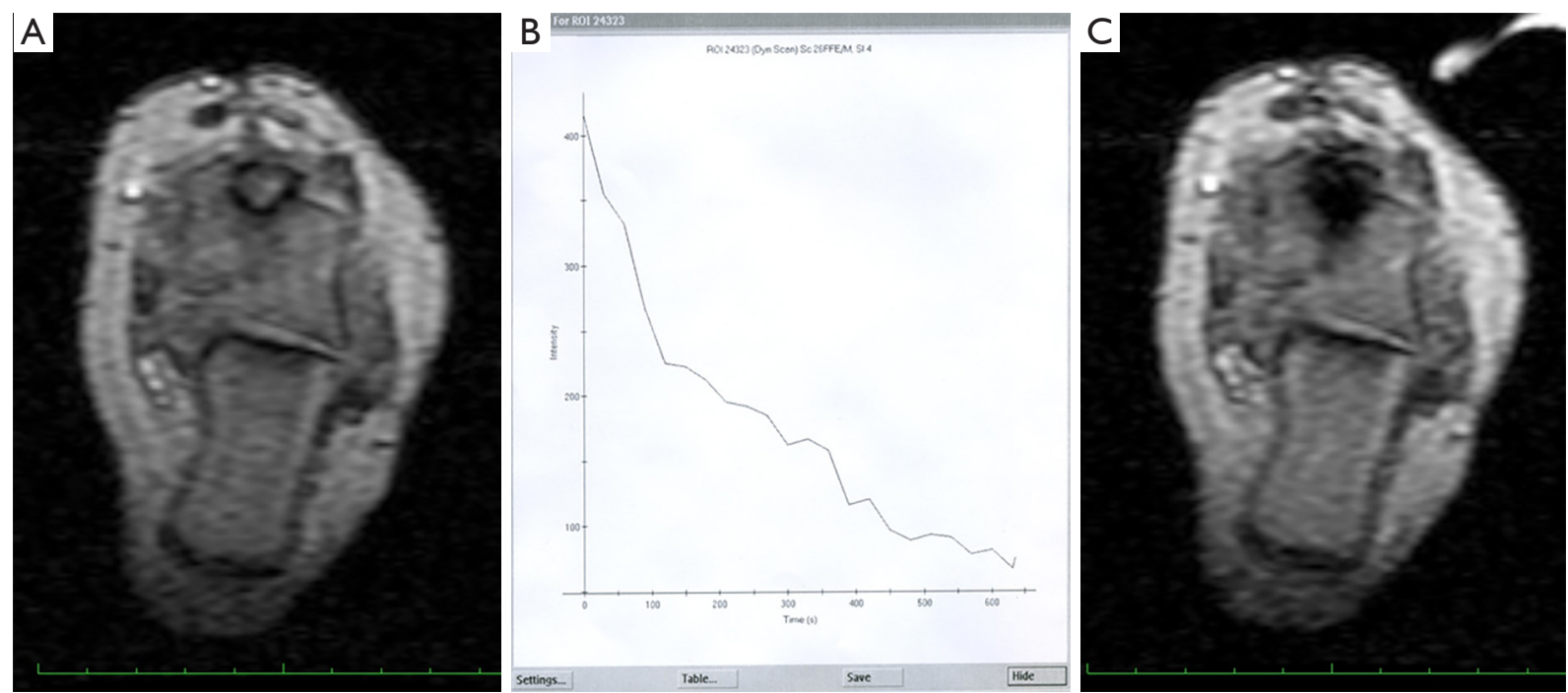

Figure 5 MRI thermometry (T1 relaxation). During the procedure, MRI thermometry was used to spare neurovascular structures in the vicinity (white circle in Figure 3C). T1-signal loss in the OO of the right talus in the patient presented in Figure 3. Images of MR thermometry (A) at beginning $(\mathrm{t}=0 \mathrm{~s})$, and $(\mathrm{C})$ at the end of laser ablation $(\mathrm{t}=600 \mathrm{~s})$ with a clear demarcation of the ablation zone, while sparing neurovascular structures. (B) Graph illustrates decrease of signal intensity over time. See Video 1. OO, osteoid osteoma; MR, magnetic resonance.

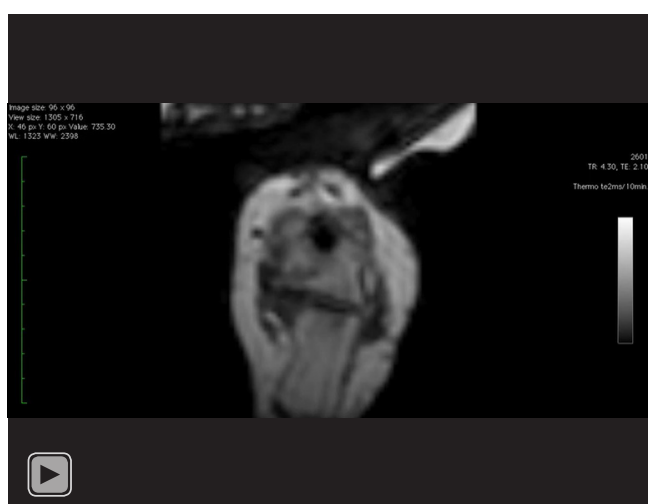

Video 1 Online MRI thermometry (T1 relaxation) during application of MR guided LA of an OO of the right talus in a 64-year-old female patient as presented in Figures 3,5. MR, magnetic resonance; LA, laser ablation; OO, osteoid osteoma.

follow-up. This also resulted in a high willingness to undergo the same procedure again, if necessary. The results in terms of satisfaction are also consistent with reports from previous studies $(6,10,41,43)$.

As limitations, the study focused on MRI-guided LA without direct comparison to CT-guided RFA. Although we conducted a telephone survey instead of a personal clinical examination, still a number of patients was lost to followup, resulting in a smaller number of patients available for long-term interviews. As strength of this study we want to underline the long follow-up period of a mean of 116 months. Long-term imaging follow-up was not conducted, which, however, is justified by the absence of clinical symptoms.

\section{Conclusions}

In conclusion, MRI-guided LA of OO is a safe and effective technique with high patient satisfaction and acceptance rates in short-term and long-term outcome. MRI thermometry can help interventionalists spare neurovascular structures in the proximity of atypically located OO. Recurrence and minor adverse events were more common in atypically located OO, suggesting that CT should be considered in patients with $\mathrm{OO}$ in locations requiring higher local resolution for image guidance.

\section{Acknowledgements}

We would like to thank Bettina Herwig for thorough language editing of our manuscript.

Funding: None. 


\section{Footnote}

Reporting Checklist: The authors have completed the MDAR reporting checklist. Available at https://atm.amegroups. com/article/view/10.21037/atm-21-3343/rc

Data Sharing Statement: Available at https://atm.amegroups. com/article/view/10.21037/atm-21-3343/dss

Conflicts of Interest: All authors have completed the ICMJE uniform disclosure form (available at https://atm. amegroups.com/article/view/10.21037/atm-21-3343/coif). The authors have no conflicts of interest to declare.

Ethical Statement: The authors are accountable for all aspects of the work in ensuring that questions related to the accuracy or integrity of any part of the work are appropriately investigated and resolved. The study was conducted in accordance with the Declaration of Helsinki (as revised in 2013). The study was approved by institutional ethics committee of Charité - University Medicine Berlin (No: EA1/301/12) and individual consent for this retrospective analysis was waived.

Open Access Statement: This is an Open Access article distributed in accordance with the Creative Commons Attribution-NonCommercial-NoDerivs 4.0 International License (CC BY-NC-ND 4.0), which permits the noncommercial replication and distribution of the article with the strict proviso that no changes or edits are made and the original work is properly cited (including links to both the formal publication through the relevant DOI and the license). See: https://creativecommons.org/licenses/by-nc-nd/4.0/.

\section{References}

1. Kransdorf MJ, Stull MA, Gilkey FW, et al. Osteoid osteoma. Radiographics 1991;11:671-96.

2. Laurence N, Epelman M, Markowitz RI, et al. Osteoid osteomas: a pain in the night diagnosis. Pediatr Radiol 2012;42:1490-501; quiz 1540-2.

3. Mungo DV, Zhang X, O'Keefe RJ, et al. COX-1 and COX-2 expression in osteoid osteomas. J Orthop Res 2002;20:159-62.

4. Mylona S, Patsoura S, Galani P, et al. Osteoid osteomas in common and in technically challenging locations treated with computed tomography-guided percutaneous radiofrequency ablation. Skeletal Radiol 2010;39:443-9.
5. Ciftdemir M, Tuncel SA, Usta U. Atypical osteoid osteomas. Eur J Orthop Surg Traumatol 2015;25:17-27.

6. Seemann RJ, Märdian S, Schwabe P, et al. Atypically Located Osteoid Osteoma: Characteristics and Therapeutic Success After Image-Guided Thermal Ablation. Rofo 2020;192:335-42.

7. Streitparth F, Gebauer B, Melcher I, et al. MR-guided laser ablation of osteoid osteoma in an open highfield system (1.0 T). Cardiovasc Intervent Radiol 2009;32:320-5.

8. Sluga M, Windhager R, Pfeiffer M, et al. Peripheral osteoid osteoma. Is there still a place for traditional surgery? J Bone Joint Surg Br 2002;84:249-51.

9. Rosenthal DI, Hornicek FJ, Wolfe MW, et al. Percutaneous radiofrequency coagulation of osteoid osteoma compared with operative treatment. J Bone Joint Surg Am 1998;80:815-21.

10. Gebauer B, Collettini F, Bruger C, et al.

Radiofrequency ablation of osteoid osteomas: analgesia and patient satisfaction in long-term follow-up. Rofo 2013;184:959-66.

11. Lindner NJ, Ozaki T, Roedl R, et al. Percutaneous radiofrequency ablation in osteoid osteoma. J Bone Joint Surg Br 2001;83:391-6.

12. Rimondi E, Mavrogenis AF, Rossi G, et al. Radiofrequency ablation for non-spinal osteoid osteomas in 557 patients. Eur Radiol 2012;22:181-8.

13. Kjar RA, Powell GJ, Schilcht SM, et al. Percutaneous radiofrequency ablation for osteoid osteoma: experience with a new treatment. Med J Aust 2006;184:563-5.

14. Shanmugasundaram S, Nadkarni S, Kumar A, et al. Percutaneous Ablative Therapies for the Management of Osteoid Osteomas: A Systematic Review and MetaAnalysis. Cardiovasc Intervent Radiol 2021;44:739-49.

15. Cantwell CP, Obyrne J, Eustace S. Current trends in treatment of osteoid osteoma with an emphasis on radiofrequency ablation. Eur Radiol 2004;14:607-17.

16. Jiang R, Jia S, Qiao Y, et al. Real-time volumetric MR thermometry using 3D echo-shifted sequence under an open source reconstruction platform. Magn Reson Imaging 2020;70:22-8.

17. Streitparth F, Walter T, Wonneberger U, et al. MR guidance and thermometry of percutaneous laser disc decompression in open MRI: an ex vivo study. Cardiovasc Intervent Radiol 2014;37:777-83.

18. Lindquester WS, Crowley J, Hawkins CM. Percutaneous thermal ablation for treatment of osteoid osteoma: a systematic review and analysis. Skeletal Radiol 
2020;49:1403-11.

19. Napoli A, Bazzocchi A, Scipione R, et al. Noninvasive Therapy for Osteoid Osteoma: A Prospective Developmental Study with MR Imaging-guided High-Intensity Focused Ultrasound. Radiology 2017;285:186-96.

20. Arrigoni F, Napoli A, Bazzocchi A, et al. Magneticresonance-guided focused ultrasound treatment of nonspinal osteoid osteoma in children: multicentre experience. Pediatr Radiol 2019;49:1209-16.

21. Maurer MH, Gebauer B, Wieners G, et al. Treatment of osteoid osteoma using CT-guided radiofrequency ablation versus MR-guided laser ablation: a cost comparison. Eur J Radiol 2012;81:e1002-6.

22. Sequeiros RB, Fritz J, Ojala R, et al. Percutaneous magnetic resonance imaging-guided bone tumor management and magnetic resonance imaging-guided bone therapy. Top Magn Reson Imaging 2011;22:171-7.

23. Sequeiros RB, Hyvönen P, Sequeiros AB, et al. MR imaging-guided laser ablation of osteoid osteomas with use of optical instrument guidance at 0.23 T. Eur Radiol 2003;13:2309-14.

24. Fuchs S, Gebauer B, Stelter L, et al. Postinterventional MRI findings following MRI-guided laser ablation of osteoid osteoma. Eur J Radiol 2014;83:696-702.

25. Dookie AL, Joseph RM. Osteoid Osteoma. Treasure Island (FL): StatPearls Publishing; 2021.

26. Wu H, Lu C, Chen M. Evaluation of minimally invasive laser ablation in children with osteoid osteoma. Oncol Lett 2017;13:155-8.

27. Tomasian A, Cazzato RL, Auloge P, et al. Osteoid osteoma in older adults: clinical success rate of percutaneous image-guided thermal ablation. Clin Radiol 2020;75:713.e11-6.

28. De Filippo M, Russo U, Papapietro VR, et al. Radiofrequency ablation of osteoid osteoma. Acta Biomed 2018;89:175-85.

29. Streitparth F, Hartwig T, Walter T, et al. MR guidance and thermometry of percutaneous laser disc decompression in open MRI: an initial clinical investigation. Eur Radiol 2013;23:2739-46.

30. Böning G, Hartwig T, Freyhardt P, et al. MR-guided lumbar facet radiofrequency denervation for treatment of patients with chronic low back pain in an open 1.0 Tesla MRI system. Ann Transl Med 2021;9:1056.

31. Perry BC, Monroe EJ, McKay T, et al. Pediatric Percutaneous Osteoid Osteoma Ablation: Cone-Beam
CT with Fluoroscopic Overlay Versus Conventional CT Guidance. Cardiovasc Intervent Radiol 2017;40:1593-9.

32. Elsholtz FHJ, Kamp JE, Vahldiek JL, et al. Periradicular Infiltration of the Cervical Spine: How New CT Scanner Techniques and Protocol Modifications Contribute to the Achievement of Low-Dose Interventions. Rofo 2019;191:54-61.

33. Yarmolenko PS, Eranki A, Partanen A, et al. Technical aspects of osteoid osteoma ablation in children using MR-guided high intensity focussed ultrasound. Int J Hyperthermia 2018;34:49-58.

34. Napoli A, Mastantuono M, Cavallo Marincola B, et al. Osteoid osteoma: MR-guided focused ultrasound for entirely noninvasive treatment. Radiology 2013;267:514-21.

35. Elhelf IAS, Albahar H, Shah U, et al. High intensity focused ultrasound: The fundamentals, clinical applications and research trends. Diagn Interv Imaging 2018;99:349-59.

36. Singh DK, Katyan A, Kumar N, et al. CT-guided radiofrequency ablation of osteoid osteoma: established concepts and new ideas. Br J Radiol 2020;93:20200266.

37. Pottecher P, Sibileau E, Aho S, et al. Dynamic contrastenhanced MR imaging in osteoid osteoma: relationships with clinical and CT characteristics. Skeletal Radiol 2017;46:935-48.

38. Kostrzewa M, Henzler T, Schoenberg SO, et al. Clinical and Quantitative MRI Perfusion Analysis of Osteoid Osteomas Before and After Microwave Ablation. Anticancer Res 2019;39:3053-7.

39. Kaptan MA, Acu B, Öztunalı Ç, et al. Correlation of 3-T MRI and CT findings with patient symptoms and treatment outcome in radiofrequency ablation of osteoid osteoma. Acta Orthop Traumatol Turc 2019;53:239-47.

40. Lassalle L, Campagna R, Corcos G, et al. Therapeutic outcome of CT-guided radiofrequency ablation in patients with osteoid osteoma. Skeletal Radiol 2017;46:949-56.

41. Beyer T, van Rijswijk CSP, Villagrán JM, et al. European multicentre study on technical success and long-term clinical outcome of radiofrequency ablation for the treatment of spinal osteoid osteomas and osteoblastomas. Neuroradiology 2019;61:935-42.

42. Hage AN, Chick JFB, Gemmete JJ, et al. Percutaneous Radiofrequency Ablation for the Treatment of Osteoid 
Osteoma in Children and Adults: A Comparative Analysis in 92 Patients. Cardiovasc Intervent Radiol 2018;41:1384-90.

Cite this article as: Seemann R, Böning G, Schwabe P, Teichgräber U, Gebauer B, Streitparth F. Osteoid osteoma: treatment outcome and long-term follow-up after MRI-guided laser ablation. Ann Transl Med 2022;10(5):240. doi: 10.21037/ atm-21-3343
43. Gebauer B, Tunn PU, Gaffke G, et al. Osteoid osteoma: experience with laser- and radiofrequency-induced ablation. Cardiovasc Intervent Radiol 2006;29:210-5. 